Historic, Archive Document

Do not assume content reflects current scientific knowledge, policies, or practices. 

ESTABLISHED /828. JANUARY ISI. 1902 INCORPORATED I895

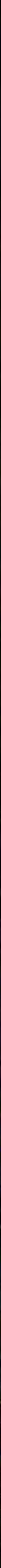




\section{Buist's Garden Seeds}

\section{IN PACKETS}

\section{The Grand Prize Medal Brand}
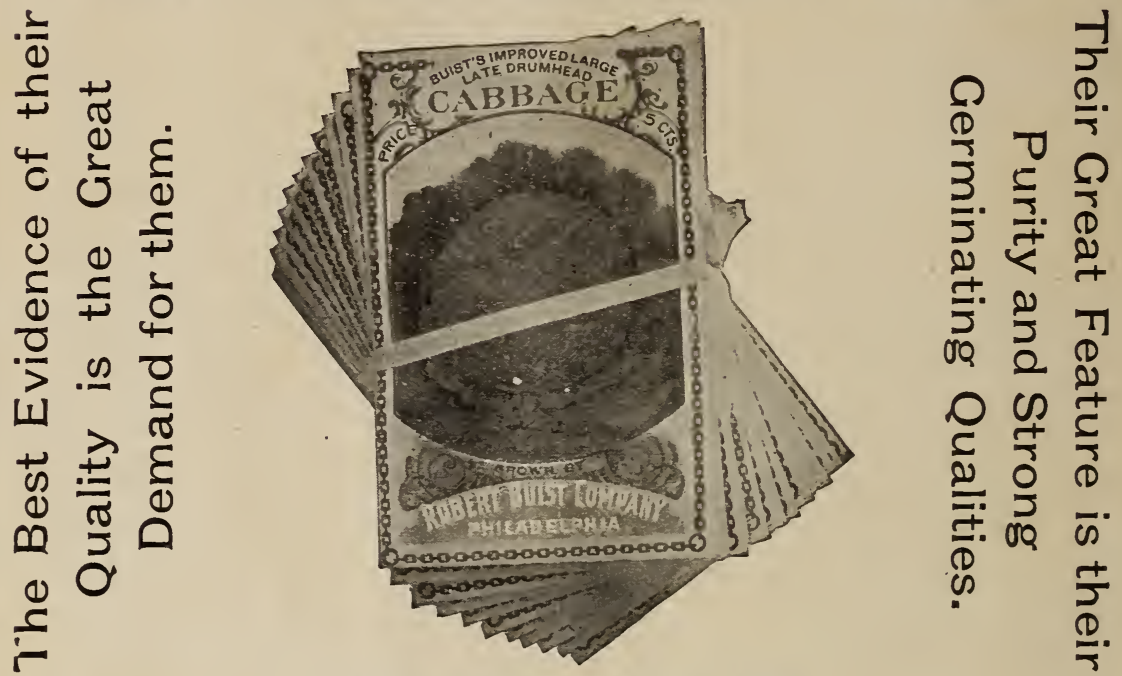

Illustration of Buist's Piotor?al Brand Packets.

PRICE FOR THE SMALL OR HALF SIZE PAPERS.

For 1,000 Papers at $\$ 10.00$

For 3,000 Papers. at

For 5,000 Papers

For 10,000 Papers

For 25,000 Papers at

REDUCED PRICE FOR THE LARGE OR FULL SIZE PAPERS.

For 1,000 Papers. at $\$ 18.00$

For $\mathbf{3 , 0 0 0}$ Papers............................................................. 17.50

For 5,000 Papers.......................................................................at 17.00

For 10,000 Papers.................................................................at 16.50

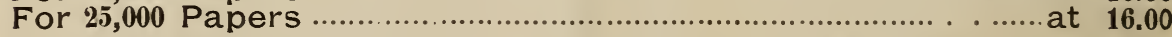

REDUCED PRICE FOR THE BUIST'S NEW PICTORIAL BRAND. Per, 1,000 Papers. at $\$ 20.0$ :

These are the most elegant and attractive Seeds in packets in the trade. They sell themselves. They have been reduced this season from $\$ 25.00$ to $\$ 20.00$ per 1,000 packets, and from $\$ 3.00$ to $\$ 2.50$ per 100 .

It is to the interest of all merchants who sell seeds to keep Buist's Growth, as no seeds are ever put up in packets but the new crop of each season, those left over from the previous year are cut out whether they grow or not. The result is our seeds will grow equally as well the second year as the first, and many will grow equally as well the third vear: such as Beet, Cabbage, Collards, Celery. Cucumber, Kale, Lettuce, Ir ustard, Parsley, Radish, Pumpkin, Squash, Tomato and Turnip, that is, of course, provided they have been properly kept from heat or dampness.

In the Southern States there are more of them sold than all other brands combined. They are of new crop each season and grown by us from selected seed stocks. There could be nothing finer produced than what we send out.

Terma of Sale.-Four months credit-cash discount, five per cent. if paid within ten davs. Accounts are subject to draft when due.

Net Cash Goods are Onion Sets, Potatoes, Bird Seed, Grass, Clorer and all other Agricultural Seeds, and must be remitted for when ordered, or be subject to sight draft on shipment.

Perishable Goods.-Onion Sets and Potatoes are al wars shipped by us in the very finest order, and packed to curry safely; but as they are subject to damage by excessive heat or cold in transit, orer which we h-ve no control, such risk must be assumed by the purchaser. No claims can be allowed for damages.

Barrels, Boxes and Sacks are charged at cost. We deliver all goods F. O. B. Philad'a.

Seed Cabinets.-Display Cabinets made of Ash at $\$ 2.00$ each. Flat Walnut Cabinets at $\$ 2.50$ each. Large Walnut Cabinets containing 49 separate drawers, 815.00 . These cabinets contain no seeds. 


\section{Buist's Seeds in Cabinet=Assortments}

Buist's

Pictorial

Brand

of Garden

Seeds in

Packets are

the most

elegant and

attractive

in the trade

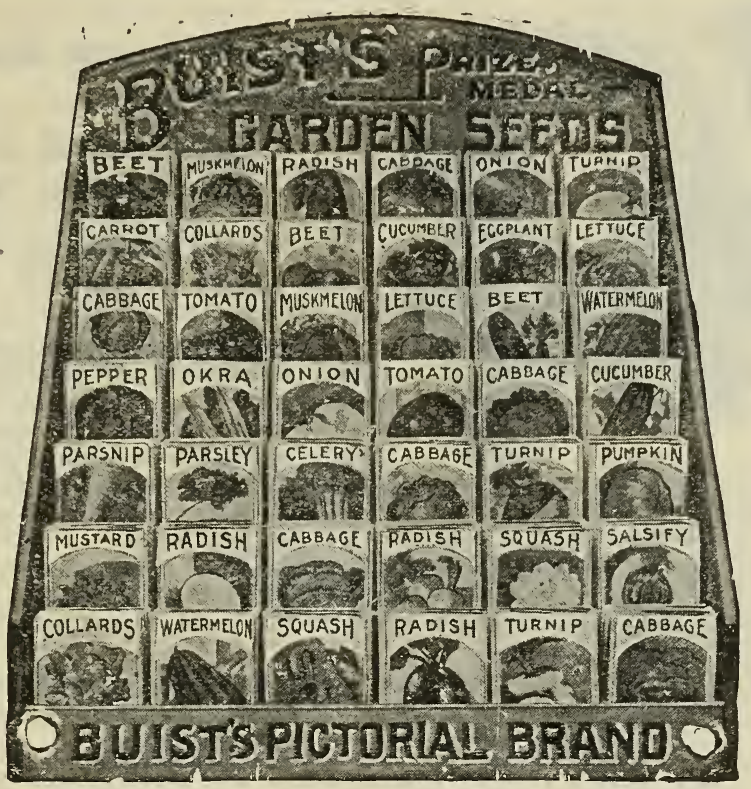

It requires

too Lalking or

persuading to

sell them, as

they sell them-

selves. It is

a well known

fact the vegetables Buist's

Seeds produce are as near per-

fection as can

be grown.

\section{CABINET ASSSORTMENTS}

No. 1 contains 500 packets Garden Seeds,

50 $1 / 3$ pints Peas, Beans and Corn,

$201 / 4$ lbs. Turnip Seeds, .

Full retail value, $\overline{\mathbf{3 4 . 0 0}}$

Price to Merchants, \$18.00. (Cabinets included.)

No. 2 contains $\mathbf{7 5 0}$ packets Garden Seeds, $\$ \mathbf{\$ 3 7 . 5 0}$

7.5 $1 / 3$ pints Peas, Beans and Corn, . . . 7.50

$251 / 4$ lbs. Turnip Seeds, . . . . . $\quad 5.00$

Full retail value, $\overline{\mathbf{5 0 . 0 0}}$

Price to Merchants, \$24.50. (Cabinets included.)

No. 3 contains $\mathbf{1 , 0 0 0}$ packets Garden Seeds

$100 \mathrm{1} / 3$ pints Peas, Beans and Corn, $\cdot \quad+\$ 1000$

40 1/4 lbs. Turnip Seeds, . . 8.00

$\frac{8.00}{68.00}$

Price to Merchants, \$32 50. (Cabinets included.)

No. 4 contains 500 packets Garden Seeds, . . . . \$25.00

No. $1 / 3$ pints of Peas, Beans or Corn, or $1 / 4$ lbs. Turnip Seeds,

Full retail value are included in this assortment.

Price to Dealer, $\$ 12.00$. (Cabinets included.)

No. 5 contains 1000 packets Garden Seeds, . . . . . \$50.00

No. $1 / 3$ pints of peas, Beans or Corn, or $1 / 41 b s$. Turnip Seeds, are included in this assortment.

Price to Dealers, \$22.00. (Cabinets included.)

BARRELS, BOXES AND SACKS are not returnable for credit, and will only be accepted to be used on future orders. 


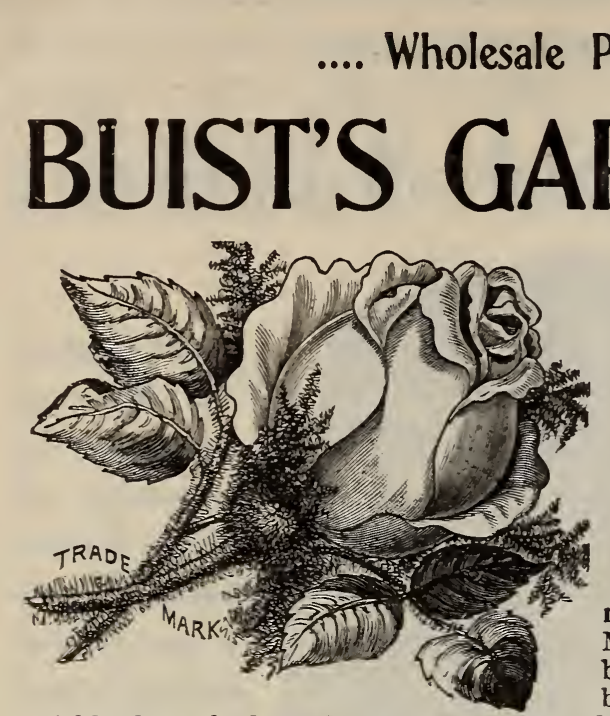

\section{Price Current ....} Grown by us from Selected
Seed Stocks.

Are celebrated for their Purity, Fine Quality, Strong Growth.

We sell 10 pounds or over at 100 pound quototion.

Your Garden Seed trade can be very much increased by handling Buist's Prize Medal Seeds ; they are not only the most Popular, but the most Sa'able. No profit is ever made by the merchant in selling cheap seeds, as he quickly loses both trade and reputation-they are either old stocks or those of an inferior quality. The market is overflowing with old bankrupt stocks, some of which are as old as our grandfather's clock; they are good enough to burn, but not to sow.

Owing to the scarcity of many varieties this season these quotations are only for prompt orders, which we will book now and ship at any date desired. If you are a large purchaser of seeds, it will afford us great pleasure to make you special quotations by letter if you will kindly enclose us a list of your requirements.

\section{BUSH BEANS-Green-Podded.}

\section{Improved Extra Early Red Speckled Valentine}

Buist's Lightning Extra Early Valentine

Early Mohawk, Six Weeks

Early Long Yellow, Six Weeks . . . Bay

Tel.

10 bush. of Per

Ballot

$\$ 3.00$

$\$ 3.25$

Babel

3.25

3.50

3.00

3.25

Refuge, or 1000 to 1

- Beadle

3.00

3.25

Extra Early Refuge

Blood

3.00

3.25

Best of All

Best of All (Buist's Selected Stock)

Band

3.00

3.25

3.75

4.00

4.00

4.25

Shipper's Favorite

Bight

4.25

4.50

Burpee's Stringless Green Pod . . . Broker

3.25

3.50

Early China Red-Eye

Bark

3.00

3.25

Early White Valentine . . . . . Barbaric

4.25

4.50

White Marrow

White Kidney .

Baron

3.00

3.25

Bedim

3.00

3.25

Henderson's Bush Lima . . . . . Butter

5.00

Burpee's Bush Lima

Buoy

5.75

5.25

6.00

\section{BUSH BEANS.-Wax or Golden-Podded.}

Dwarf German or Black Wax .

Bait

3.75

4.00

Dwarf German Black Wax (Buist's Selected Stock) Bare 
BUSH BEANS-Wax or Golden-Podded-Continued.

Currie's Rust-proof Black Wax .

Dwarf Prolific Black Wax

Dwarf Golden or Cream Wax

Dwarf Golden or Cream Wax (Buist's Selected)

Wardwell's Speckled Kidney Wa

Dwarf Crimson Wax Flageolet

Dwarf Scarlet Market Garden Wax

Dwarf Butter Wax Bismarck .

Dwarf White Wax

Keeney's Rustless Golden Wax

Valentine Wax

Refugee Wax

Detroit Wax

Perfection Wax

\section{BEANS.-Pole.}

Large White Lima

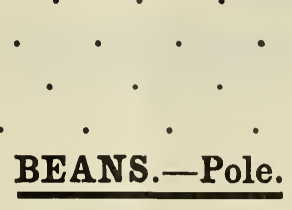

Salem Mammoth Lima

Extra Early Jersey Lima

King of the Garden Lima

Ford's Mammoth Podded Lima

Carolina, Sieva, or Small Lima.

Dreer's Improved or Challenger Lima

German, or Black Wax Pole

Southern Prolific

Improved Large Podded Southern Prolific.

Kentucky Wonder, or Old Homestead

Southern Crease-Back, or Fat Horse .

Golden Cluster Wax Pole

Golden Wax Flageolet Pole

Lazy Wife's Pole

Horticultural, or Wren's Egg

Scarlet Runners

White Dutch Case Knife

Red Speckled Cut-Short or Corn Hill
Yosemite Mammoth Dwarf Wax

Tel.

Cipher.

10 bush. of Per

Brunt \$3.7.5 \$400

Bedrench 400

4.25

Bake

3.75

4.00

Buzz.

4.00

4.25

Bluff

4.25

4.50

Brisk

4.00

4.25

Border

4. 25

4.50

Bugle

4.25

4.50

4.00

4.25

7.00

Becoming

Bellows

400

4.25

Below

4.00

4.25

Beside

4.00

4.25

Benzine

4.00

Binder

4.25

4.25

4.50

5.00

5.25

Bedeck

5.50

5.75

Boggle

5.75

6.00

Bray

5.75

Beholden

5.50

5.75

Binnacle

5.50

6.50

Bismuth

6.25

5.75

Brain

5.50

5.75

Bode

5.50

5.50

4.25

550

Banquet 4.00

5.25

5.50

Birdlime 5.2.5

5.50

6.00

5.50

6.25

4.50

4.25

5.75

Brier

5.25

Brave

6.00

5.50

Bright

5.25

4.50

4.25

Broth

5.25

5.50

PEAS. Crop very short, espec:aily Early Varieties.

The Buist Morning Star Peas, which we introduced a few years since, is the earliest and most productive variety known, and is regarded by Market Gardeners as their best and must profitable shipping variety. For protection to ourselves and to insure the purchaser true stock, we sell them only in our Sealed Packages and Lead Sealed Sacks, each Seal is sta mped Buist. The latter are of green muslin and contain $1 / 8,1 / 4,1 / 2$. or one bushel each. Its great popularity has caused almost ever dealer to catalogue them and a cheap grade of Extra Earlies is substituted. Observe, unless they are put up in green sacks and lead sealed with our name they are spurious.

Buist's Extra Early

Pate

4.00

4.25

Buist's Premier Extra Early (Sealed)

Pack

4.25

4.50 
PEAS.-Continued.

Tel. Cipher.

10 bush of

Buist's Early Morning Star (In green sacks, sealed) Pathos $\$ 4.50$

First and Best (New York Stock)

Pang

4.00

Buist's First and Best

Page

4.00

Extra Early Market

4.25

Extra Early Hancock

Park

4.00

Extra Early Dandy

Pass

4.00

First of All

Prefer

4.25

Early Alaska

Paint

Philadelphia Extra Early

Portly

5.25

Pail

4.00

Rural New Yorker

4.00

Blue Beauty.

Purvey

5.25

Improved Early Dan O'Rourke .

Purloin

4.00

Pave

Improved Early Dan O'Rourke (Selected Strain) Pounce

4.00

Early Washington

4.00

Early Frame

Pawn

4.00

Early May .

Peace

4.00

Carter's Daisy .

Pearl

Pouch

French Canner

Petal

3.50

Extra Early Premium Gem

Poise

5.50

McLean's Little Gem .

Horsford's Market Garden

Plod

5.50

Early American Wonder

Puff

3.75

Plain

5.75

Nott's Excelsior

Pencil

6.00

Champion of England

Prey

3.50

Forty Fold

Press

3.50

Yorkshire Hero .

Pride

3.50

Early Tom Thumb .

Plate

4.25

Gregory's Surprise

Eugenie .

Port

Dwarf Blue Imperial .

Prank

4.00

Prism

3.25

Prow

4.25

Per

$\$ 4.75$

Telephone

Improved Stratagems .

Shropshire Hero

Purple

4.00

4.25

4.25

4.50

4.25

4.25

4.50

5.50

4.25

4.25

5.50

4.25

4.25

4. 25

4.25

4.25

7.00

3.75

5.75

5.75

4.00

6.00

6.25

3.75

3.75

3.75

Duke of Albany .

Parish

375

450

7.00

4.25

3.50

4.50

Pastel

4.25

4.25

Heroine. .

Prefix

3.50

4.00

Gradus

Laxton's Evolution

Purity

4.50

Pride of the Market

Potion

4.25

3.75

Improved Pride of the Market

Punch

4.00

10.00

Telegraph (Long Island Mammolh)

Prussic

4.25

4.50

4.25

Pause

3.25

4.50

Bliss's Everbearing .

Pottage

3.50

3.50

Bliss's A bundance

Public

3.50

McLean's Advancer

Plume

350 
PEAS.-Continued.

Black-eyed Marrowfat (Hand Picked)

Tel.

Cipher.

Prose

10 bush, of Per a variety Bush. $\$ 2.35$ $\$ 2.50$ Black-eyed Marrowfat (Not Hand Picked)

Pestle

2.25

2.40

Large White Marrowfat (Hand Picked)

Prune

2.35

2.50

Large White Marrowfat (Not Hand Picked)

Poniard

2.25

2.40

Royal Dwarf White Marrow

Piston

2.35

2.50

Mammoth Podded Sugar .

Post

Dwarf or Tall Sugar (Edible Pod)

Peep

Prudent

5.25

6.00

5.50

6.00

ARTICHOKE.

Green Globe

Abate

Per 100 lbs. Per $1 b$. 225.00

2.50

1.50

Jerusalem Artichoke Roots

Per bushel

ASPARAGUS.

Large Purple Top

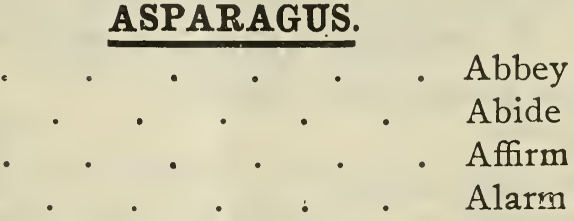

Abbey

24.00

.28

24.00

.28

Barr's Mammoth

Affirm

24.00

24.00

.28

.28

ASPARAGUS ROOTS.

Conover's Colossal ( Two Years Old)

Agrail

Per 100 Per 10000

.50

4.00

.60

4.50

The Palmetto (Two Years Old)

Albino

.50

4.00

\section{BEET.-Our Choicest Selected Stock.}

Extra Early Turnip or Bassano .

Blind

Per I00 1bs. Per 1b.

20.00

.25

Early Red Turnip .

Birch

20.00

Early Dark Blood Turnip (Selected)

Bit

25.00

.25

Extra Early Egyptian Red Turnip.

Boot

25.00

Crosby's Egyptian

Brass

Extra Early Egyptian Red Turnip (Buist's Stock) Big

30.00

.30

.30

Early Eclipse Red Turnip.

Bobbin

28.00

30.00

Bless

25.00

Dewing's Early Red Turnip .

Bud

22.00

Blot

45.00

Buist's Extra Early Red Turnip

Bastian's Early Red Turnip

Boat

20.00

Lentz's Early Red Turnip

Bold

22.00

.50

.25

Edmand's Blood Turnip

Butment

Bend

25.00

.25

.30

Early Othello Blood Turnip

Columbia or Excelsior

Brigade

35.00

.40

Boxer

35.00

Stinson's Early Red Turnip

Brewery

25.00

Detroit Dark Red Turnip .

Boston

30.00

Electric

Booth

28.00

28.00 


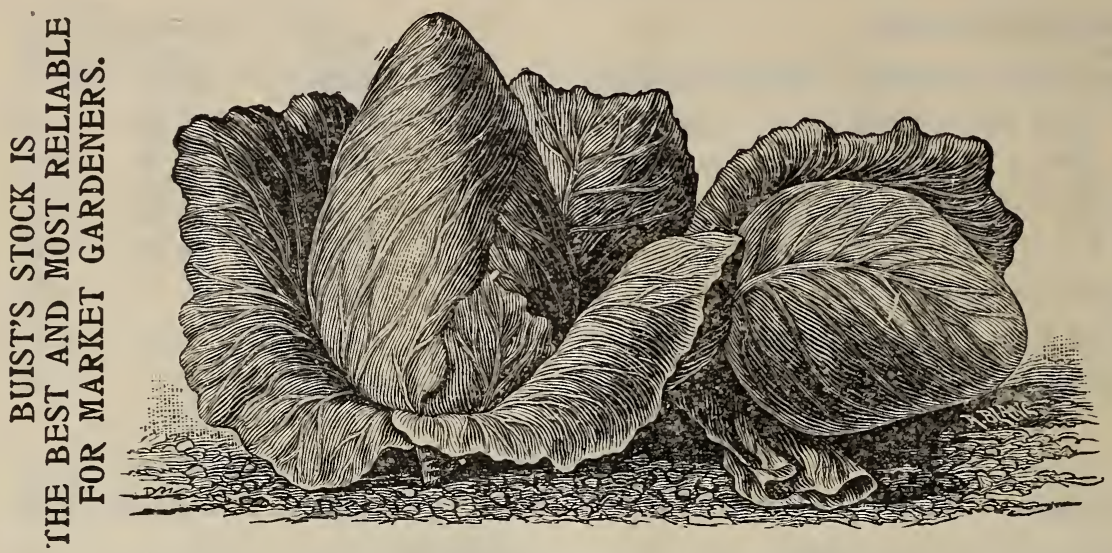

BUIST'S EXTRA EARLY JERSEY WAKEFIELD CABBAGE. Its Great Features are its Size and Earliness.

BEET.-Our Cholcest Selected Stock.-Continued.

I-1ong Blood Red

Buist's Improved Long Blood

Bastian's Half-long Blood .

$\begin{array}{ccc}\text { Tel. } & \text { Per } \\ \text { Cipher. } & 100 \text { lbe. }\end{array}$

Per lb.

Beguile $\$ 20.00$

$\$ .25$

Beaten $\quad 30.00$

.35

Bedaub

22.00

\section{BEET.-Mangolds or Feeding Varieties.}

White French Sugar . . . . . . Befall

15.00

.25

Lane's Imperial Sugar . . . . . Bias

15.00

.18

Giant Long Red Mangel . . . . Beach

1200

.18

Orange Globe Mangle

Brow

1200

.15

Champion Mammoth Long Red Mangle . Balm

1200

.15

Champion Yellow Globe Mangel . . . Brink

12.00

.15

Buist's Mammoth Long Red Mangel . . Bolt

15.00

.15

Buist's Golden Globe Mangel . . . . Burr

1500

.20

Golden Tankard Mangel . . . . Bandage

12.00

.20

Chirk Castle Mammoth Red Mangel . . Bloom

35.00

.15

Norbiton's Giant Mangel

Brocade

12.00

.15

\section{BROCOLI.}

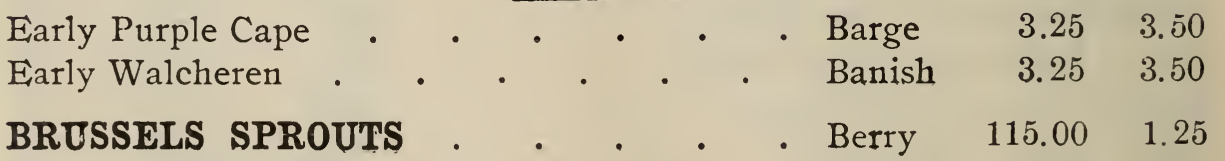

\section{CABBAGE.}

All varieties of Cabbage Seed sold by 119 are grown from our speclal seed stocks, which are famous for their great purity and certainty of heading.

Early Large York . . . . . . Coy 70.00

Buist's Early Large York . . . . Covert 100.00

Early Jersey Wakefield (Selected American)

Cynic 150.00

1.60

Early Jersey Wakefield (Imported)

Cygnet 110.00 


\section{THE THREE GREAT VARIETIES OF CABBAGE FOR THE SOUTH.}
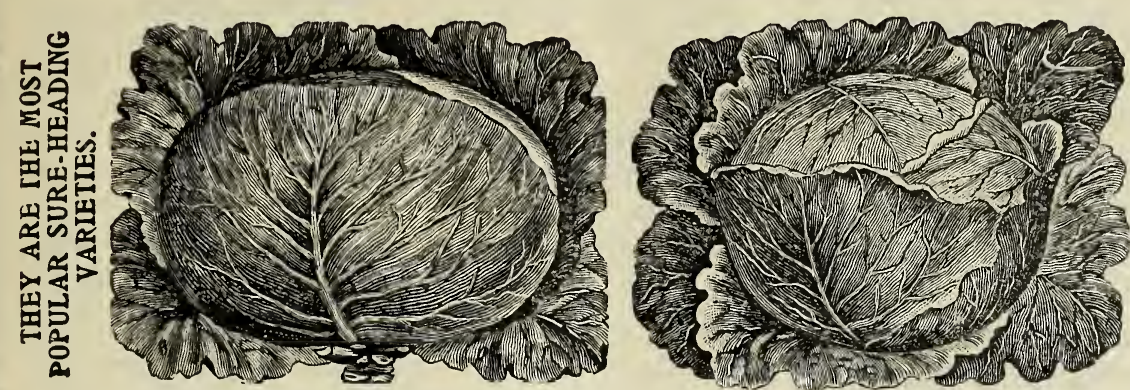

BUIST'S IMPROVED LARGE DRUMHEAD CABBAGE.

BUIST'S IMPROVED LARGE FLAT DUTCH.

BUIST'S FLORIDA HEADER.

\section{CABBAGE.-Continued.}

Buist's Extra Early Jersey Wakefield (Sealed)

The Charleston, or Large Jersey Wakefield

The Charleston Wakefield (Imported) . .

Henderson's Early Summer

Early Summer, (Imported)

Henderson's Succession

Early English Winnigstadt

Early French Winnigstadt

Extra Early Express

Early Etampes

Buist's Earliest

All Head Early

Long Island Large Late Drumhead . .

Long Island Large Late Flat Dutch . . .

Premium Large Late Drumhead

Premium Large Late Flat Dutch

Large Late Drumhead

Large Late Flat Dutch

Buist's Improved Late Drumhead (Sealed),

(The Prize Medal Stock) .

Buist's Improved Late Flat Dutch (Sealed),

(The Prize Medal Stock) .

Market Gardeners' Late Flat Dutch.

Buist's Florida Header (Sealed), (Prize Stock)

Buist's Short-Stem Brunswick Drumhead

Buist's Early Flat Duch (Sealed)

Buist's Early Drumhead (Sealed)

Improved Early Flat Dutch
Tel.
Cipher

Car

Per

$100 \mathrm{lbs}$.

$\$ 175.00$

Per lb.

$\$ 2.00$

Caravan 160.00

1.75

Chronic 150.00

1.60

Cancel 150.00

1.60

Clipper 100.00

1.10

Cycle

160.00

1.75

Casper

95.00

1.00

Candie 115.00

1.25

Cartridge 140.00

1.50

Cathedral140.00

1.50

Convey 160.00

1.75

Colony $2(10.00$

Cruet

115.00

2.10

1.25

Caw

115.00

Cube

110.00

1.25

1.25

Cup

110.00

Classify 110.00

1.25

1.25

1.25

Claimant 110.00

2.00

Creed 175.00

2.00

1.50

2.25

2.00

2.00

2.00

1.25 
CABBAGE.-Continued.

Improved Early Drumhead Stein's Early Flat Dutch

Early Dwarf Flat Dutch

Early Dwarf Drumhead

Louisvile Drumhead

Large Surehead .

All Seasons (American)

All Seasons (Imported)

The Lupton

The Vandergaw .

North Carolina Buncombe

Autumn King or World Beater

Danish Ball Head or Amager.

Short-Stem Drumhead

Hollander or German Export .

Fottlers Improved Brunswick

Marblehead Mammoth

Green Glazed

Red Dutch

Blood Red Berlin

Large Red Drumhead

Mammoth Red Rock .

Improved Red Stone-head

\section{CABBAGE, SAVOY.}

Green Curled Savoy

Cement 110.00

American Curled Savoy

Censer

110.00

Green Globe Savoy

Chat

110.00

Large Drumhead Savoy

Cling

110.00

Crisp

110.00

Per lb.

$\$ 1.25$

Perfection Drumhead Savoy

CARROT. Crop very short.

Early French Horn

Early Parisian Forcing .

Early Scarlet Horn

Guerande, or Ox-heart

Early Half-long Scarlet (Pointed Root).

Early Half-Long Scarlet (Stump Root)

Danver's Half-long Scarlet

Buist's Half-long Scarlet (Pointed Root)

Saint Vallery or Scarlet Intermediate

Half-long Chantenay

Half-long Nantes

Half-long Luc
Cincture 60.00

Citadel $\quad 65.00$

Chuck 60.00

Cogitate 70.00

Connect 65.00

Cross

Churn

65.00

65.00

Codicil $\quad 55.00$

Climax $\quad 65.00$

Clarionet 65.00

Cruise

65.00
1.25

1.25

1.25

1.60

1.10

1.10

1.20

140

1.60

1.25

1.50

160

1.25

1.50

1.8 .5

1.40

1.85

1.10

120

1.35

1.25

1.50

1.25

1.50

1.50

1.25

1.25 
CARROTS.-Continued.

Half-long Carentan

Long Orange

Buist's Improved Long Orange .

Large White Belgian

\section{CAULIFLOWER.}

Early London

Tel.

Cipher.

Per

Clergy

65.00

Per lb.

Comply

55.00

$\$ .70$

Canine

55.00

.60

Check

28.00

.60

.30

Extra Early Paris

Convey

Per oz.

Half Early Paris

Chief

.50

4.00

Coop

.60

6.00

6.00

Early Walcheren

Choir

.50

3.50

Veitch's Autumn Giant

Chime

3.00

Le Normand Short Stem

Church

5.50

Large Early White Erfurt

Copy

1.00

14.00

Extra Early Dwarf Erfurt

Chimney

1.60

20.00

Buists Prize Early Snowball

Chin

1.60

22.00

Large Algiers .

Crown

.50

6.00

CELERY.

Large White Solid .

Churl

Per 100 ibs.

Buist's Large White Solid

Cider

90.00

1.00

Golden Dwarf .

Circle

120.00

1.25

75.00

.80

Dwarf Golden Heart .

Cape

85.00

1.00

Golden Yellow Self-blanching

City

240.00

2.50

Golden Self-blanching (highest grade)

Contract 325.00

3.40

Improved White Plume .

Corps

110.00

1.25

Giant Pascal

Perfection Hartwell

Clap

65.00

.75

Crum

90.00

1.00

Sandringham Dwarf White

Clank

80.00

.90

Boston Market

Kalamazoo Broad Ribbed .

Classic

75.00

.80

Convent 100 0C

1.10

Colossal 110.00

1.25

Dwarf Pink, or Rose

Celeriac, or Turnip-rooted Celery

Soup, or Flavoring Celery

Coral

90.00

1.00

Cosse?

10.00

CHERVIL.-Double Curled

(-)

Cadge

50,00

COTLA RDS. WE MAKE the growing of Coli Jr' Seed a specialty, and annually gcll more seed than any (n) other house in the trade. It 3 acknowledged that we have the best heading White Stem Cabbage Collards known in the South. The greatimportence of securing a fine strain is well known by every Southern merchant, as it is one of their most imp ortant crops. The cheap Seed offered by the trade is imported from England and is more of a Kale or VVil ? Cabloge chan Ooldards, and causes great dissatisfaction, Georgia (Selected White Stem)

Southern Blue Stem .

Southern Creole

North Carolina Buncombe

Buist's Cabbage Collards

Camus 50.00

.55

Cadis

50.00

Callot

60.00

.55

60.00

\section{CORN SLAD}

Large Seeded

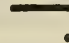

Canyon

70.00

Capos 


\section{CORN.-Garden Varieties.}

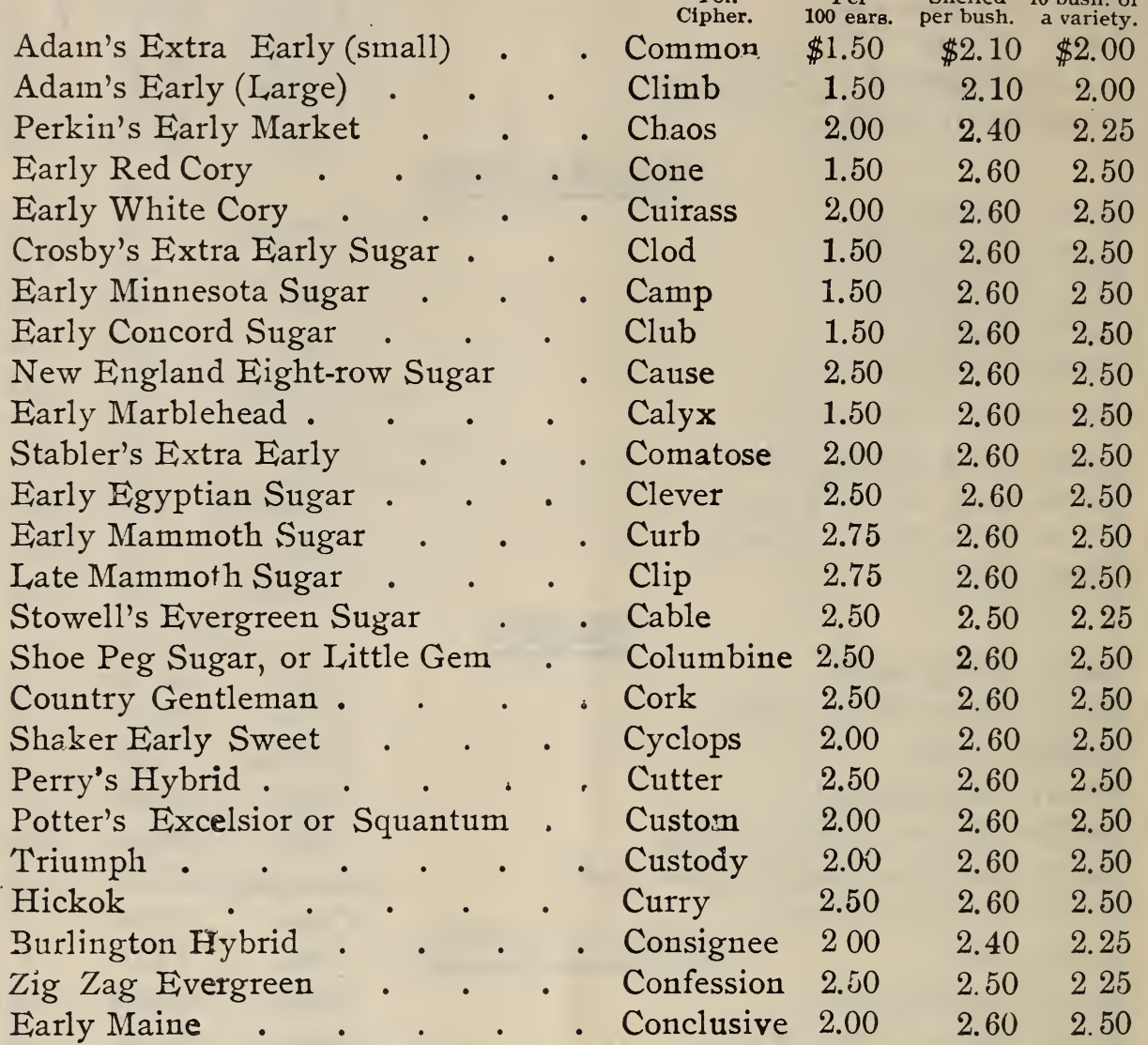

\section{CORN.-Field Varieties.}

All our Sheiled Corn is from selected ears, the small points being taken off before shelling.

Early Golden Dent

Criminal

2.00

1.25

1.20

Early Golden Dent (Selected) . . Clef

2.00

1.35

1.30

Buist's Improved Early Golden Dent

Cotton

2.00

1.50

1.40

(The best Yellow Field Corn for the South.)

Buist's Prize Medal Southern Snow Flake

(The Best White Field Corn for the South)

Canoe

2.00

1.50

1.40

The above tivo varieties are only sold in our lead sealed 2-bushel sacks, each seal stamped Buist.

Buist's Large White Flint, or Hominy, Cod

Improved Large Maryland White . Comb

2.00

1.50

1.40

White Hickory King . . . Comedian

2.00

1.50

1.40

Improved Early Leaming .

2.00

1.50

1.40

Golden Beauty

Crook

2.00

1.50

Courtly

2.00

1.50

1.40

Mammoth Eared Gourd Seed . . Coil

Chester County Gourd Seed . . Color

2.50

1.50

1.40

Cloud's Early Dent Gourd Seed

2.00

1.50

1.40

White Cap Dent

Combat

2.00

150

1.40

Clique

2.00

1.50

1.40

1.40 

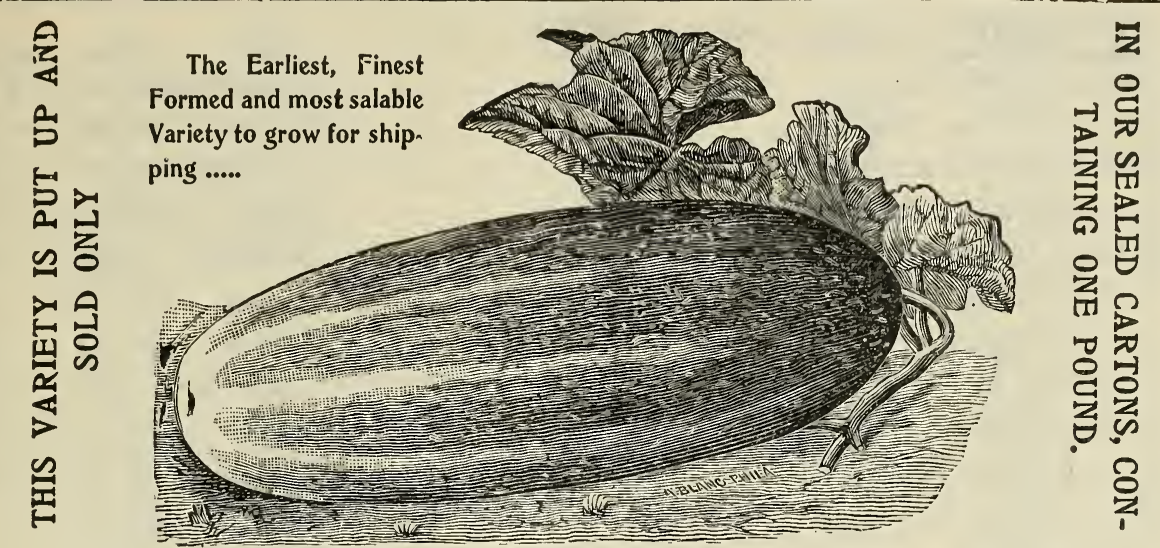

BUIST'S PERFECTION EARLY WHITE SPINE CUCUMBER.

The Great Market Garden Variety.

CORN

N. - F Field Varieties.-Continued. Blunt's White Prolific - Clatter

Per

Shelled 10 bush, of Early Yellow Canada Complex $\$ 2.00$ per bush. a variety Early Pennsylvania eight-row . . Cord

2.00 $\$ 1.50$ $\$ 1.40$ Champion White Pearl . . . Curdie 2.00 150 1.40 1.50 1.40 Large White Dent . . . . Cupola 2.00 1.50 Mastodon Yellow Dent . Cupel

2.00

1.50

1.40

1.40

2.00

1.50

CRESS, or PEP PER GRASS.

Curled Che

Tel.

Per

$1001 \mathrm{~b}$.

Water

Carbon

20.00

Carlic 200.00

Per $1 \mathrm{~b}$

\section{CUCUMBER.}

Early White Spine

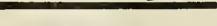

Improved Early White Spine or Arlington

Arlington or Early White Spine (Western Grown.)

Carmot

24.00

Cartel

30.00

Cress

25.00

Buist's Perfection Early White Spine (Sealed),

Evergreen White Spine.

Casing

35.00

Cordage

25.00

Evergreen White Spine, (Western Grown.).

Cupid

25.00

.40

Peerless White Spine

Cracker

25.00

Hill's Forcing White Spine

25.00

Thorburn's Everbearing .

Console

35.00

.30

Casque

25.00

Early Frame

Cavern

25.00

Early Cluster

Caxon

25.00

Early Russian

Census

Cephus

25.00

Long Green

Chela

25.00

London I tong Green

Cess

30.00

Long Green Turkey

Ceroon

35.00

Buist's Long Green

Canty

45.00

Westerfield's Chicago Pickling

Crosier

25.00 


\section{CUCUMBER.-Continued.}

Boston Pickling

New Orleans Market.

Fordhook Pickling .

Livingstone's Evergreen

Giant Pera

Tailby's Hybrid .

Jersey Pickle .

Nichol's Medium Green

Green Prolific Pickle

Gherkin, or Burr

White Wonder

White Pearl

Cool and Crisp

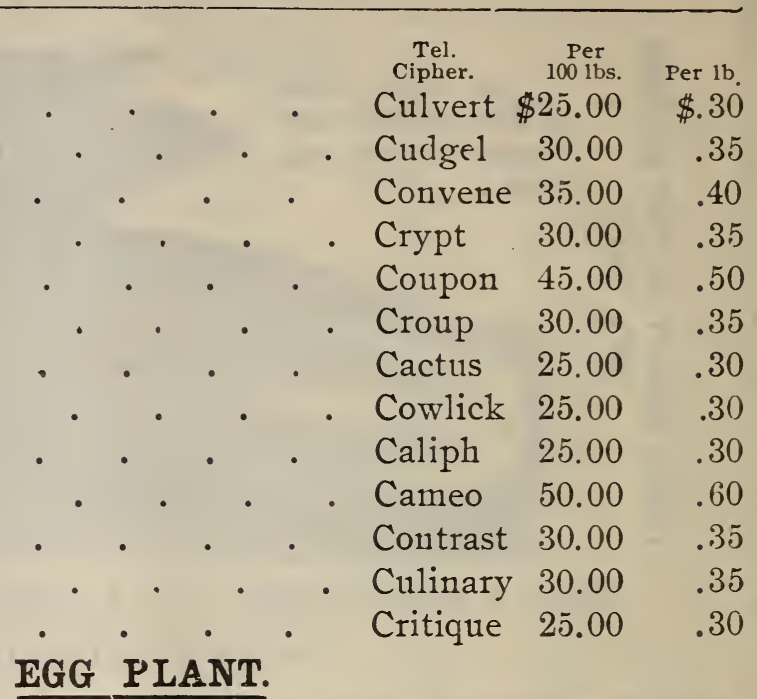

Most of the Egg Plant Seed sold by the trade is of Southern growth, which, owing to poor seed stock, produces imperfect fruit, both in form and color. The seed which we offer is of our own growth (we annually grow 25 acres), and there is no finer stock known than the Buist's Improved, which is the best market variety.

New Jersey Improved Large Smooth • • . Ease 190.00

2.00

Large New York Improved Purple . . . Echo 190.00

Buist's Improved Large Thornless Purple . . Edge 250.00

2.60

(Largest and Finest Fruit and best market variety ; color-dark, rich, purple.)

\section{ENDIVE.}

Mammoth Green Curled . • • • . Eagle 90.00

1.00

Green Curled Winter.

White Curled .

Enrage

70.00

Ebony

70.00

Broad Leaved Batavian

Earl

70.00

Market Gardeners Green Curled

Enigma 120.00

\section{KALE}

Dwarf Green Curled Scotch

Philadelphia Green Curled Scotch

Tall Green Curled Scotch

Dwarf German Greens

Siberian Curled

Norfolk Curled .

New Intermediate

Mosbach Curled Winter

New Dwarf Erfurt .

Dwarf Curled Berlin .

Spring Kale or Sprouts

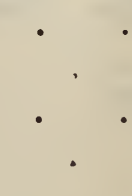

Kedge

45.00

1.25

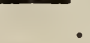 \\ .}

Kindred 45.00

.50

.50

Knight

45.00

.50

Keel

45.00

.50

Kesar

30.00

.35

Knack $\quad 45.00$

Knel

60.00

Kimbo

60.00

.65

Ken

60.00

Knout

60.00

Kernel

10.00

KOHL RABI.

Large Green, or White

Key

80.00

Early White Vienna (Short-leaved)

Khan

140.00

.90

Earliest White Vienna (For Frames)

Kirtle

160.00

1.50

Early Purple Vienna

Kieve $\quad 150.00$ 
LEEK

London Broaf Flag . . . . . . Lack

Tel.
Cipher
Lack
Loop

Per

100 lbs. Per $1 \mathrm{~b}$.

Large American Flag

Lad

$\$ 60.00$

Buist's Mammoth

Large Musselburgh

Lair

70.00

$\$ .65$

Monstrous Carentan

Laky

90.00

.75

1.00

Large Rouen.

Lamb

80.00

.90

80.00

LETTUCE.

75.00

Philadelphia Early White Cabbage or Butter

Lance

40.00

Early Prize Head

Lath

40.00

.50

Early Curled Silesia

Lasso

40.00

.45

Early Curled Simpson

Larva

40.00

Black Seeded Simpson

Latin

45.00

Improved Early Hanson

Lavish

45.00

Early Tennisball (White Seeded)

League

55.00

Early Tennisball (Black Seeded)

Lathe

50.00

Buist's Perfection White Forcing

Levee

125.00

Early Boston Market

Early Speckled Dutch Butter

Legal

55.00

Leper

65.00

San Francisco Passion

Link

75.00

American Royal Cabbage

Letch

45.00

Improved Royal Cabbage

Ledme

60.00

Lieve

60.00

Black Seeded Royal

Henderson's New York

Lantern

70.00

Latitude

55.00

Big Boston

Limpet

75.00

Big Boston (Selected) .

Laundry

90.00

Premium Cabbage .

The Deacon Cabbage .

Lateral

70.00

Oak Leaved

Larynx

55.00

Legible

75.00

Denver Market

Lenient

50.00

Golden Standwell

Liberal

90.00

Stubborn Head

Liturgy

90.00

Black Seeded Butter

Drumhead Cabbage

Lottery

45.00

Lilac

45.00

Brown Dutch (Black Seed)

Limbo

60.00

Perpignan, or Early Summer

Limit

70.00

White Paris Cos (Romaine)

Lintel

Lisp

55.00

California Cream Butter

Loathe

50.00

Salamander

Lengthy

55.00

Blonde Blockhead

Golden Stonehead

Ladle

55.00

.45

.45

.50

.50

.60

.55

1.40

.60

.70

.80

.50

.65

.65

.75

.60

.80

1.00

.75

.60

.80

.60

1.00

1.00

.50

.50

.70

.75

.60

.55

.60

.60

Lignite

55.00

Iceberg

Lowery

55.00

.60

Silver Ball

Lumber

55.00

All the Year Round

45.00

\section{MELON.-Cantaloupe or Musk.}

Netted Green Citron . $\cdot$ • $\cdot$ • 
MELON.-Cantaloupe or Musk.-Continued.

Rocky Ford (Finest Selected).

Tel.

Netted Nutmeg

Chicago Market Nutmeg

Hackensack, or Turk's Cap

Extra Early Hackensack

Miller's Cream, or Osage

Emerald Gem

Acme, or Baltimore Citron

Jersey Belle

Pine Apple

Early Jenny Lind.

Netted Gem (Round) .

Netted Gem, (Oblong)

Champion Market

Paul Rose

Bay View

Montreal Market

New Orleans Market .

Washington Market

Delmonico .

Banquet

Extra Early Cape May

Mountain Sweet

\section{MELON.-Water.}

Kolb's Gem

Georgia Rattlesnake, or Striped Gypsy

Augusta Rattlesnake (Selected)

Duke Jones

The Dixie

Jordan's Gray Monarch

Florida Favorite

Girardeau's Premium Triumph

Ice Cream (White Seeded)

Ice Cream (Gray Seeded)

Phiney's Early

Round Light Icing, or Ice Rind

Long Light-Icing

Dark Icing

Seminole

Cuban Queen

Peerless

Pride of Georgia

Sweet Heart

Blue Kolb Gem .

Bradford .

Mammoth Iron Clad

Black Boulder

Green Preserving Citron

Cipher.

Per

Martial $\$ 35.00$

Per 1b.

Magic $\quad 30.00$

.40

Minaret 30.00

.35

Myth

30.00

.35

Mattock 3500

.35

Mediate 3500

.40

Meridian 35.00

40

Meteor 30.00

.40

Marsh $\quad 30.00$

.35

Magnet 30.00

.35

Maim $\quad 25.00$

.35

Mastic

3.5 .00

Maul

3500

Minimum 35.00

.30

.40

Manicure 35.00

Mango 30.00

.40

.40

.40

Mantel $\quad 30.00$

.35

Maple $\quad 75.00$

.35

Moat $\quad 75.00$

.80

Metaphor 35.00

Melt

35.00

.40

Money $\quad 30.00$

.35

March $\quad 20.00$

.25

Marble $\quad 18.00$

Mast 24.00

.28

Mead

30.00

.35

Mania 28.00

.32

Motor

22.00

.25

Model $\quad 30.00$

.35

Manes 28.00

.32

Moist 45.00

.50

Merge 25.00

.30

Mécal $\quad 25.00$

.30

Monody 25.00

.30

Mica $\quad 25.00$

.30

Majestic 30.00

.35

Mild

25.00

Mink

30.00

Mitre

22.00

Moan

24.00

Mode $\quad 24.00$

Monk 24.00

.30

.35

Manklnd 25.00

.28

.28

Manner 45.00

.30

Merrily 25.00

.50

Mettle $\quad 30.00$

Mock 


\section{MUSHROOM SPAWN.}

Finest English Milltrack in Bricks 500 lbs. and over at 6 cts. per $1 \mathrm{~b}$.

Tel.
Cipher.

Per

100 lbs. Per lb.

\section{MUSTARD.-For Growing.}

White London Myrrh

$\$ 7.00$ $\$ .08$

Black or Brown Mope

12.00

Mammoth Souther 'jurled Moral

12.00 Ostrich Plume

Chinese

MUSTARD.-For Flavoring.

Molar $\quad 5000$

Molest $\quad 50.00$

Merino $\quad 60.00$

White London

Mound

8.00

Black or Brown

Musk

8.00

NASTURTIUM, or Indian Cress.

Tall Yellow (For Pickling)

Notion $\quad 50.00$

Dwarf Tom Thumb (Fine Varieties)

Neutral 40.00

Tall Mixed (Fine Varieties)

Near

40.00

\section{OKRA or GUMBO.}

Buist's Dwarf Green Prolific

Oasis

25.00

Perkin's Mammoth Podded

Oath

25.00

Tall, or Long Green

Obey

20.00

White Velvet

Object

25.00

Dwarf White .

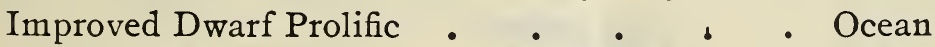

25.00

.25

.30

.30

Lady Finger

Ode

25.00

25.00

.30

.30

ONION

Large Yellow Round Danvers

Orris

60.00

Large Yellow Globe Danvers .

Obscure 70.00

Buist's Yellow Globe Danvers

Oven

75.00

Philadelphia Yellow Dutch or Strasburg

Oblong

65.00

Large Red Wethersfield

Oblate

75.00

Australian Brown

Oxide

75.00

Philadelphia White, or Silver Skin . . . Observe 150.00

South port Yellow Globe .

Outwit

80.00

South port Red Globe .

Osier

80.00

Southport White Globe .

Ochre

150.00

Extra Early Red

Oriole

70.00

.65

\section{ONION. - Italian and Bermuda Varieties.}

Giant Red Bermuda

- Olive

160.00

Improved Red Bermuda Our Bermuda Onion Seed . Omen

175.00

1.80

Giant White Bermuda

Owl

Improved White Bermuda

Obese

175.00

1.80

185.00

Mainmoth Silver King

Ordeal

150.00

1.60

Mammoth Yellow Prizetaker . . . . Opaque

95.00

1.00

Giant Yellow Rocca

Giant Red Rocca

Opera

90.00

1.00

Giant White Tripoli

Office

90.00 
ONION.-Italian and Bermuda Varieties.-Continued.

Early White Barletta.

Early White Queen

Early White Pearl

Orator $\$ 165.00$

$\$ 1.7 \%$

Organ 150.00

1.60

Offer $\quad 165.00$

1.75

\section{BUIST'S SELECTED ONION SETS}

We are the most extensive growers and shippers of Onion Sets in the trade, with a reputation of shipping the finest samples. They are smaller, more uniform in size, and better cleaned than those of Western growth, and will keep longer without sprouting. We also use a $7 / 8$ mesh in cleaning them, while Western and many other growers use an inch, making our sets of much smaller size and more salahle. We fan and clean each order separately, Onion sets are sold for net cash and subject to cost for barrels and crates for packing them

\&o These quotations are for immediate orders, and for shipment during January or February as required, for these months we supply $351 \mathrm{bs}$. to the bushel, and not 32 as are given by Western houses

Yellow or Red

White or Silver Skin .

Dark Red Wethersfield
Per bushel of $3 \tilde{j}$ pounds.

646

$16 \quad 66 \quad 66$

\section{PARSLEY.}

Finest Double Curled.

Emerald or Dwarf Extra Curled

Buist's Finest Selected Moss Curled.

Champion Moss Curled

Extra Dark Moss Curled

Plain, or Single

\section{PARSNIP.}

Sugar or Hollow Crown

Improved Griernsey

Student

\section{PEPPER}

Bell, or Bull Nose

Large Sweet Spanish

Golden Dawn

Ruby King

Squash, or Tomato

Long Red Cayenne

Red Cherry

Yellow Cashaw, or Crook-Neck

\section{PUMPKIN. \\ PIIIN}

Omega

Orbit

Onyx

Per Bush. $\$ 2.25$

3.00

2.50

Per $100 \mathrm{lb}$. Per $1 \mathrm{~b}$.

Potash $\quad 30.00$

.35

Partner

35.00

Panel

35.00

.40

Parse

35.00

Prance

35.00

Path

20.00

.40

.40

.40

.25

\section{Green Striped Cashaw}

Peak

20.00

Prolific

20.00

.25

Peel

20.00

\section{Golden Marrow}

Large Cheese

Large Tours, or Mammoth

Mammoth King

Jonathan

Tennessee Sweet Potato

Nantucket

Quaker Pie

Connecticut Field

Phaca 110.00

1.25

Pesky 110.00

Phiz $\quad 140.00$

1.25

$1 ., 0$

Pecan 140.00

1.50

Pendant 125.00

1.50

Phrase 125.00

1.50

Perna $\quad 140.00$

1.50

Pew $\quad 30.00$

Product 30.00

Profit

30.00

Pied

30.00

Purse

65.00

Puzzle

60.00

Prelude

35.00

Pursue

45.00

Punt

40.00

Pupa

40.00

.45

Pur

12.00 
French Grown, Finest Stocks.

\section{RADISH.}

Early Long-Scarlet (Short Top)

Tel.

Cipher.

Race

Regnant 35.00

$\$ 32.00$

Rhyme

35.00

Raise

35.00

Review

40.00

Early French Scarlet Turnip

Range

40.00

Ransom

40.00

Relapse

40.00

Road

40.00

Russet

40.00

Rasp

40.00

Ratio

40.00

Early Scarlet French Breakfast

Early French Scàrlet, or Cardinal Globe

Early Prussian Scarlet Globe (Buist's Stock).

Wood's Early Frame

Early Deep Scarlet Olive-Shaped

French Half-Long Deep Scarlet

Extra Early White Turnip

Early White Turnip

Philadelphia Early White Box

Charter, or Shepherd

Improved Yellow Summer 'Turnip

Improved White Summer I'urnip

Golden Globe Summer

Long Black Spanish (Winter)

Round Black Spanish (Winter)

Chinese Rose (Winter)

White Summer Strasburg

Long White Vienna, or Lady Finger

Buist's Long White

Celestial, or New White Chinese
Rout

40.00

Rifle

Rejoice

60.00

32.00

Raven

40.00

Ravine

Reach

40.00

35.00

35.00

Roster

35.00

Rugged

35.00

Rebuke

35.00

Recede

35.00

Record

35.00

Reign

30.00

Rector

30.00

Reduce

25.00

Ruby

40.00

Revoke

Relic

35.00

Rescue
35.00

Per $1 \mathrm{~b}$.

$\$ .35$

.40

.40

.40

.45

.45

.45

.45

.45

.45

.45

.45

.45

.65

.35

.45

.45

.40

.40

.40

.40

.40

.40

.40

.35

.35

.30

.45

.40

.40

.45

\section{RHUBARB.}

Large Victoria

Rural

75.00

.80

Ruler

75.00

SALSIFY, or Oyster Plant.

Long White American

Serve

75.00

Mammoth Sandwich Island

Saxon

90.00

Buist's Mammoth White

Sage

115.00

1.00

1.25

300 lbs. at 10 ets. per. 11).

SPINACH.

$5001 \mathrm{lbs}$ at 9 ets. per $1 \mathrm{~b}$.

Buist's Perfection Curled (Finest Curled)

American Curled Savoy

Scoff

12.00

Salam

12.00

.14

Saline

12.00

Norfolk Savoy-Leaved

Sally

12.00

Round Savoy-Leaved

Sapid

10.00

Sash

12.00

.14

.14 
SPINACH.-Continued.

Enkhuizen Long Standing

Tel.

Cipher

Monstrous Viroflay (Thick-Leaved) . . Studio

Per

$1001 \mathrm{bs}$.

Scale $\$ 12.00$

Per 1b.

Scan

9.00

$\$ .14$

Prickly Seeded

New Victoria

Savor

12.00

.12

9.00

.14

SQUASH.

Early White Bush, or Patty-Pan . . . Scarce

32.00

.12

Extra Early White Bush

Scion

2.00

.35

Stroll

32.00

.35

Mammoth White Bush

Science

32.00

.35

Scrap

32.00

Yellow Summer Crook-Neck

Sharp

32.00

Giant Yellow Summer Crook-Neck

Sedan

30.00

Buist's Improved Marrow

Scope

45.00

.35

.35

.35

.35

Script $\quad 55.00$

.50

Scramble 60.00

Chicago Warted Hubbard

Shoal $\quad 38.00$

.60

.65

Marblehead

Spectrum 45.00

.40

London Vegetable Marrow

Spherics 50.00

Fordhook

Sponsal 90.00

Mammoth Chili

Shirk

30.00

.00

Orange Marrow

Squadron

.35

White Pineapple

Scrag

\section{TOMATO.}

The growing of the Tomato especially for seed, is one of our specialties. Our stocks are unequalled for their great purity and fine quality, which is the result of making an annual selection from which we grow our seed.

Buist's Beauty \} These two varieties are unequalled for shipping, Tendon 185.00

Buist's Belle $\}$ canning, or private growing. Tocsin 225.00

2.00

Buist's Selected Trophy

Teak 150.00

2.50

Buist's Selected Early Acme

Tamp 150.00

160

Buist's Selected Paragon

Livingstone's Perfection

Tardy

1.60

Temple 110.00

1.60

I-ivingstone's Favorite

Trench 110.00

1.25

Livingstone's Beauty .

Tangent 125.00

1.25

Livingstone's Stone

Tempt

1.40

Early Acme

Tame

110.00

1.25

Paragon

The Queen

Tape

125.00

1.40

Tawny

110.00

1.25

Trophy

Taunt

110.00

1.25

The Matchless

Tear

12500

1.40

Mikado

Trait

165.00

1.75

Train

125.00

1.40

Essex Hybrid

Cumberland Triumph

Tease

125.00

1.40

Early Ruby

Tint

110.00

1.25

Tower

135.00

1.40

Dwarf Champion

Thesis

200.00

2.25

Atlantic Prize

Tierce

110.00

1.25

Ponderosa

Tendril 125.00 
TOMAT0.-Continued.

Golden Trophy

Large Yellow

Red Pear-shaped

Yellow Plum-shaped

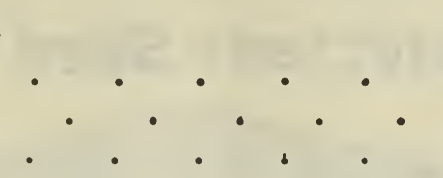

Tel.

Cipher.

Per

Tenor $\$ 150.00$

Per lb.

Tepid $\quad 150.00$

Terso $\quad 160.00$

$\$ 1.60$

Text $\quad 190.00$

1.60

1.75

2.00

Buist's Majestic (This is the largest and finest Tomato in cultivation).

In packets only, per dozen pkts., $\$ 1.00$; per hundred pkts., $\$ 7.00$.

\section{TURNIP.-White Varieties.}

We are very extensive growers of Turnip Seeds, it being one of our specialties, and can supply large orders at lower prices than any house in the trade. Our seed is grown from selested and transplanted roots and is celebrated for its great purity and fine quality. Special prices for large orders.

Red, or Purple Top Flat

Theme 20.00

.22

Red, or Purple Top (Strap-Leaved)

Thick

20.00

.22

Early White Flat Futch .

Thirst

20.00

.22

Early White Flat Dutch (Strap-Leaved)

Thorn

20.00

.22

Red, or Purple Top White Globe

Thread

24.00

.26

Buist's Mammoth Purple Top Globe .

Thrift

24.00

.26

Large White Globe

Throb

18.00

.20

Large White Norfolk .

Throng 18.00

.20

Pomeranean White Globe

Tide

18.00

Southern Snow White Globe

Timid

20.00

Early White Egg

Tinge

32.00

Large Cow Horn

Tinsel

32.00

Large White Hanover . . . . . Toast

20.00

Sweet German

Token

20.00

Southern Seven Top (For Winter Greens) . Tonic

18.00

Southern Prize (For Turnip or Winter Greens), Torch

20.00

Dixie Land (For Turnip or Winter Greens) . Turn

20.00

Extra Early Red Top Milan

Topaz

35.00

\section{TURNIP.-Yellow Varieties.}

Purple Top Yellow Aberdeen .

Yellow Stone.

Golden Bail

Large Amber Globe

I بarge Yellow Globe
Trace

22.00

Tray

Treble

22.00

22.00

Trend

20.00

Tress

\section{TURNIP, RUTA BAGA.}

Improved American Purple-Top Yellow .

Buist's Improved Purple-Top Yellow . . Bloomsdale Swede .

Tube

24.00

Tweed 24.00

Twig

24.00

Improved Long Island Purple-Top Yellow

Twitch

24.00

Sutton's Champion Purple-Top

Tall

Monarch or Elephant

Tusk

Tact

20.00

.22

Purpie Top Yellow (Imported)

22.00

Improved Hardy

Tassel

20.00

.22

Tyrant

20.00 


\section{Buist's Selected Seed Potatoes,}

\section{Grown . •}

Expressly .

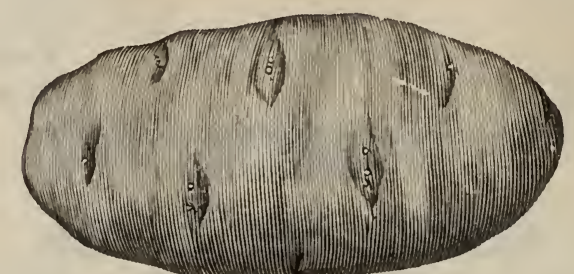

For

Planting . .

Packed in large full-sized Barrels or Barrel Sacks, containing 23/4 Bushels, 165 lbs.

THE

HE Buist Brand of Seed Potatoes have an established reputation throughout the Southern States for their Great Purity, Fine Quality and Productiveness.

They are grown in Maine, Vermont, Nova Scotia and Prince Edward's Island especially for seed, and are of an entirely different quality from the stocks generally sold, which are frequently mixed varieties of a very inferior grade, with the name branded on the barrels to suit the order. These prices are for immediate orders booked with us now, and for shipment during December or January.

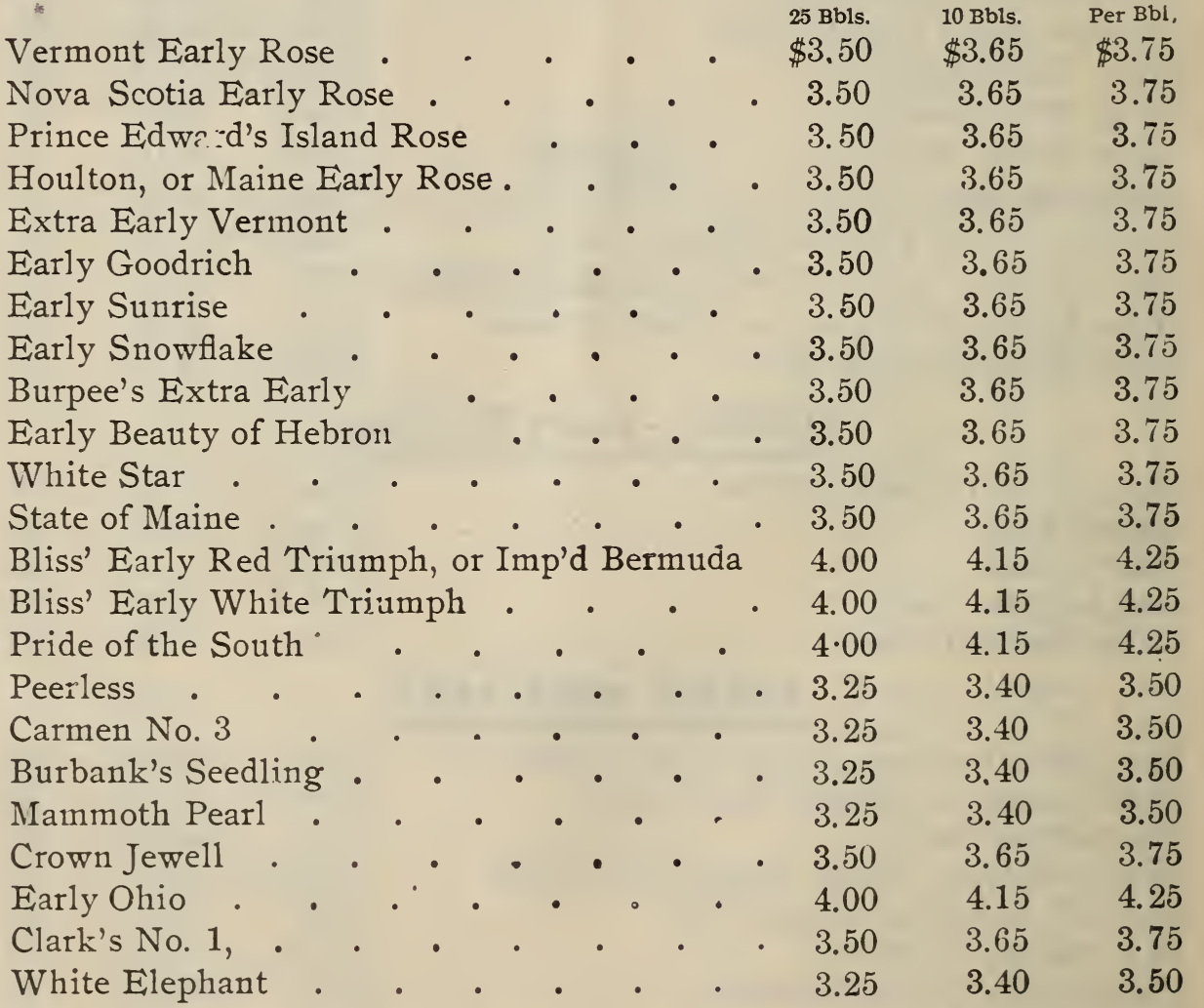

Potatoes are sold for net cash; remittanee with order or subject to sight-draft on date of shipment. Crop very short. Prices subject to change. 


\section{Buist's Named Varieties}

\section{LARGE FLOWERING SWEET PEAS.}

Alice Eckford ; creanı-tinted standard wlite wings. . $\$ 0.25$

America ; brightest blood-red striped Apple Blossom ; bright rosy pink

Aurora ; orange salinon

Blanche Burpee; largest pure white Blanclie Ferry; pink and white

Blushing Beauty ; finest delicate pink Boreatton ; deep maroon

Butterfly; white edged with lavender

Captain of the Blues; bright blueis'1 purple .

Captivation ; light magenta or claret Countess of Aberdeen; shaded pink

Countess of Radnor; pale mauve or lavender

Cardinal; bright scarlet

Cupid Dwarf White ; pure white, grows only six inches high

Cupid Dwarf Pink; rose pink, white wings, six to eight inches high

Dorotliy Tennant; deep rosy mauve

Duchess of Edinburgh; scarlet and crimson

Duchess of York; white suffused with pink

Duke of Clarence ; brilliant rosy claret

Eliza Eckford; rosy pink and white

Emily Eckford; heliotrope .

Emily Henderson; purest white

Empress of India ; white and rose

Firefly; brilliant scarlet

Gaiety ; white rose stripe

Gray Friar ; purple white ground

Her Majesty; bright rosy pink .

Ignea ; intense scariet

Indigo King ; blue and purple .

Katharine Tracy ; bri'liant pink
Per 1b.

.30

.30

.35

.40

.30

.30

.25

.30

.30

.86

Lady Beaconsfield; salmon pink and yellow .

Per $1 b$.

Lady Penzance ; pale rose

Lemon Queen ; blush, tinted lemon

Little Dorrit; pink standard white wings

Lottie Eckford; white suffused with lavender blue

Lovely ; soft shell pink

Mars ; bright scarlet

Meteor ; orange salmon

Miss Hunt ; carmine pink

Monarch; deep maroon and dark blue

Mrs. Eckford ; delicate primrose $\quad .35$

Mrs. Gladstone ; delicate pink . . . 30

Mrs. Jos. Chamberlain; white striped pale rose

Mrs. Sankey ; white

Orange Prince; orange pink . . $\quad .30$

Ovid; rosy pink .

Prima Donna; lovely shade of soft pink

Primrose ; primrose yellow . . .30

Princess Beatrice ; carmine rose $\quad .30$

Princess May; light lavender . . .30

Princess of Wales; striped mauve on white ground .

Queen Victoria; primrose shaded purple .

Rising Sun ; orange rose and carmine

Royal Robe ; delicate pink shaded

Royal Rose ; deep rosy pink .

Senator; chocolate striped white

Splendor; rich rose shaded crimson

Stanley; deep maroon

Venus; salmon buff shaded rosy pink

\section{MIXED VARIETIES OF SWEET PEAS.}

Per 1b. Per $100 \mathrm{lb}$.

Fine Mixed Varieties; $\quad \begin{aligned} & \$ 0.20 \\ & \$ 16.00\end{aligned}$

Choicest Mixed Varieties ; . . .25

Buist's Special Prize Mixture; .35
20.00

30.00
Eckford's Fine Mixed;

Per 1b. Per $100 \mathrm{lb}$

Eckford's New Hybrids; .

.35

$\$ 20.00$

French Beauties; 


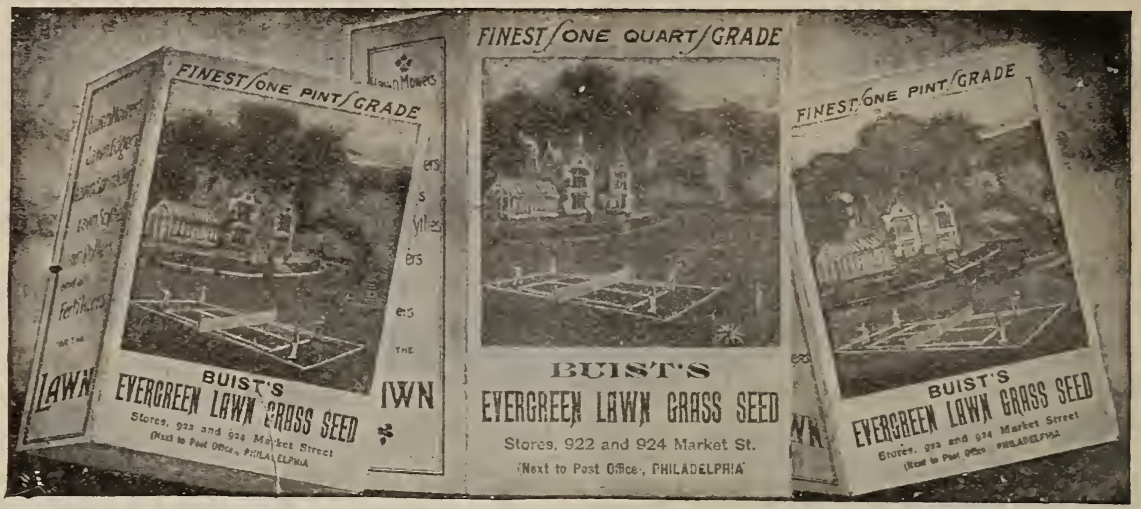

\section{BUIST'S LAWN GRASS SEED}

PUT UP IN CARTONS READY FOR RETAIL TRADE.

IS VERY SALABLE BECAUSE IT GROWS QUICKLY.

In Pint Cartons which retail at 15 cents each .........................................\$1.00

In Quart Cartons which retail at 25 cents each......................................... 1.50

In Two Quart Cartons which retail at 50 cents each.................................... 3.00

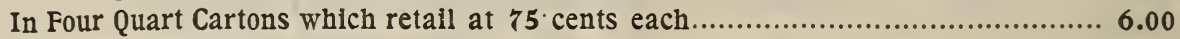

The Buist's Mixture of Grass Seeds for Lawns, Parks, Tennis and Cricket Grounds has long been famous for producing a succession of verdure throughout the year. It is composed of the finest varieties of grasses, each of which has its season of beauty, and the result of this blending is the producing of a sod which is not only always evergreen and velvety in appearance, but of the color and beauty of an emerald. Many of the more famous lawns of this country were seeded down with the Buist's Lawn Grass, the preparation of which is a specialty with our house, which many years of experience has suggested; it requires from 4 to 5 bushels to sow an acre, or for renovating old lawns about half that quantity.

Buist's Evergreen Lawn Mixture (Finest Grade), • " " \$3 \$0 Buist's Evergreen Lawn Mixture (Choice Grade), . • " 300 Buist's Fairmount or Central Park Mixture (Finest Grade), " 300 Buist's Fairmount or Central Park Mixture (Choice Grade), " $\quad 250$ Buist's Golf Greens Mixture (Finest Grade), " " 350

VARIETIES OF GRASS SEEDS. I.bs. Per

Herds, or Red-Top Grass . (Agrostis Vulgaris), . $10 \quad \$ 080$

Kentucky Blue Grass . (Poa pratensis) Fancy Cleaned, $\begin{array}{rr}14 & 165\end{array}$

Kentucky Blue Grass . (Poa protensis) Fancy Export, $14 \quad 200$

Pennsylvania Green Grass (Poa pratensis) • . . $14 \quad 190$

Creeping Bent Grass . ․ (Agrostis stolonifera), . $\quad 20 \quad 350$

Rhode Island Bent Grass $\quad$ (Agrostis Canina), . $\quad \begin{array}{ll}2 & 1250\end{array}$

Orchard Grass

(Dactylis glomerata), . 12

Timothy - . . • (Phleum pratense), . . 45

Tall Meadow Oat Grass : (Avena elatior), . . 10

Yellow Oat Grass . . (Avena flavescens), . . 7

English Perennial Rye Grass (Lolium perenne), . . 24

Italian Rye Grass . : (Lolium Italicum), . 22

Johnson Grass • • • (Sorgum Halapense) . 25

Sweet Vernal Grass (Anthoxanthemum odoratum), Per 1b.

Meadow Foxtail

(Alopecurus pratensis), . " "

Wood Meadow Grass - . (Poa nemoralis), - • • "

215

325

185

400

200

200

250

Bermuda Grass

(Cynodon.Dactylon), 


\section{VARIETIES OF CLOVER.}

American Red (Choicest recleaned), . . . . $\$ 1300$

White Dutch (American),

White Dutch (Imported) Finest Grade, . $\quad 2200$

Crimson, or Trifolium incarnatum,

\section{VARIETIES OF MILLET.}

German, or Golden M11let, Tennessee German Millet, . . . . . • . . 165 Hungarian Grass, or Millet, . . . . . . . 140 Imported French Millet, per $100 \mathrm{lbs}$., $\$ 6.00$; per $1 \mathrm{~b}$., . . . 06 Pearl, or Cat-Tail Millet, per 100 lbs., $\$ 10.00$; per lb., . . . 12

\section{Dwarf Essex Rape, Finest Imported Seed} Per 100 lbs. $\$ 8.00$; Per 1b., 10 cts.

\section{Black-Eyed Cow Peas (Whip-poor-will.)}

THE FINEST AND MOST PRODUCTIVE VARIETY

Per Bushel \$2.50. 10 Bushels at $\$ 2.40$ per Bushe1

\section{Hairy Vetch (Vicia Villosa)}

Per 100 lbs., $\$ 10.00 \quad$ Per lb., 12 cts.

\section{Buist's Prize Collections OF SUMMER BLOOMING \\ E 1}

\section{IN BEAUTIFULLY POLISHED OAK CABINETS}

These Cabinet Assortments contain only the most attractive and salable varieties, which are put up in beautifnlly lithographed packets with flowers illustrated in natural colors. They are quick sellers at 100 per cent profit. The 5 cent varieties are charged at $21 / 2$ cents each, and those sold at 10 cents, at 5 cents. They are of new crop each season and will grow perfectly well the second year.

Price with Cabinet

Collection No. 1 contains $\underset{25}{150}$ packets $\underset{10}{5}$ cent varieties,

Collection No. 2 contains 200 " 5 " 50 "

Collection No. 3 contains $\left.\begin{array}{rrrrr}150 & 6 & 5 & 6 & 6 \\ 100 & 6 & 10 & 6 & 6\end{array}\right\} \quad 9.75$

\section{SWEET AND MEDICINAL HERBS}

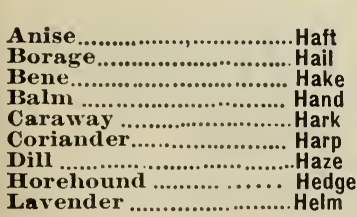

Per oz
$\$ 010$
10
15
15
6
6
10
20
15
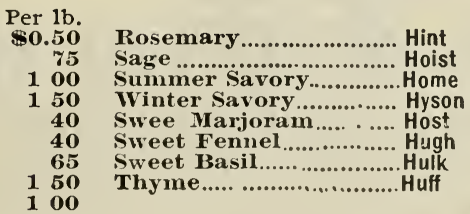

Per oz. Per $1 \mathrm{~b}$

Ta 20

20
15
15
20
15
6
15
20




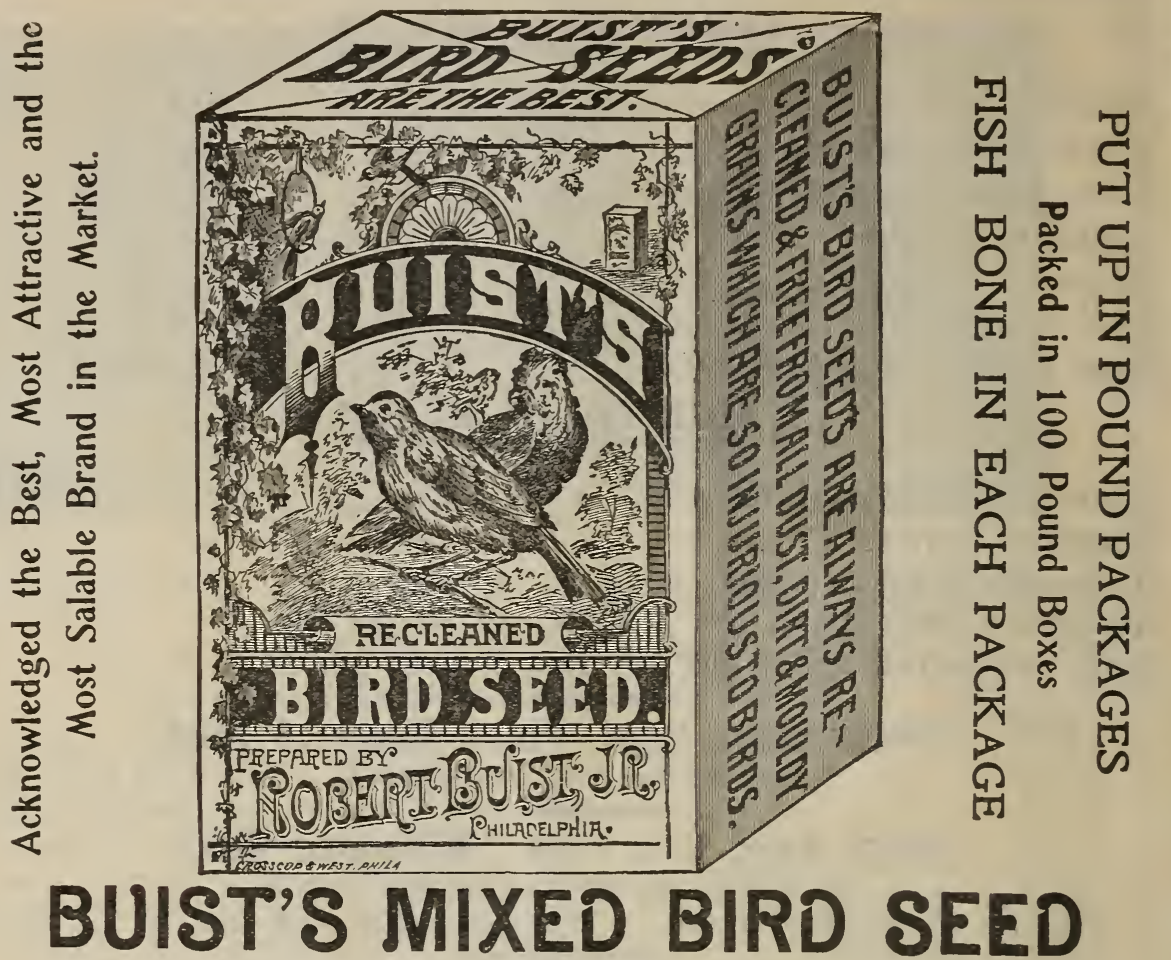

The great advantage in handling this brand is, that it has been thoroughly recleaned before being prepared and is composed entirely of the very finest grade of Seeds, some of
which is found in no other brand.

Boxes of $100 \mathrm{lbs}$. at $6 \mathrm{cts}$. per $\mathrm{lb}$., or 5 boxes at $5 \mathrm{I} / 2$ cts. per $\mathrm{lb}$. F. O. B. and no charge for Boxes.

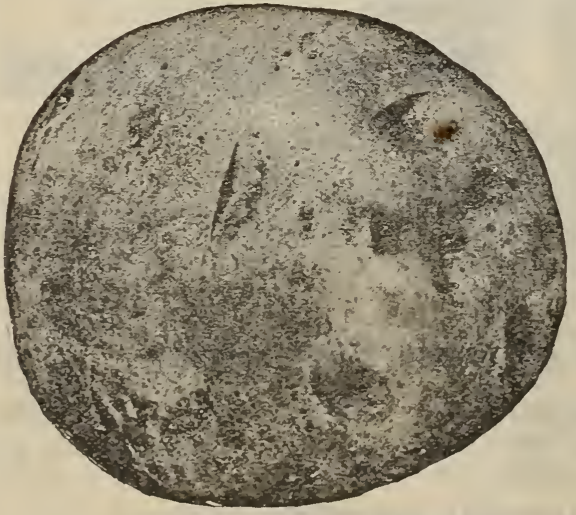

Bliss' Early Red Triumph Potato

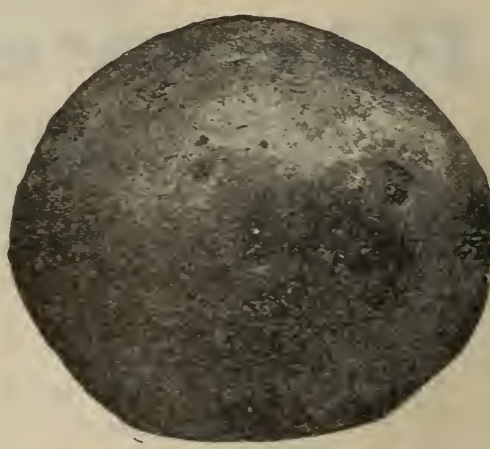

Pride of the South Bliss' Early White 'Triumph Potato.

\section{BUIST'S SELECTED SEED POTATOES Are the Finest for Your Trade}

- Merchants who Sell Seed Potatoes and desire the Choicest Quality are invited to try those put up by our house and known in the South as the Buist Brand. They are grown g'y us in Naine and other desirable locations and are the finest Stocks in the Market. 7 


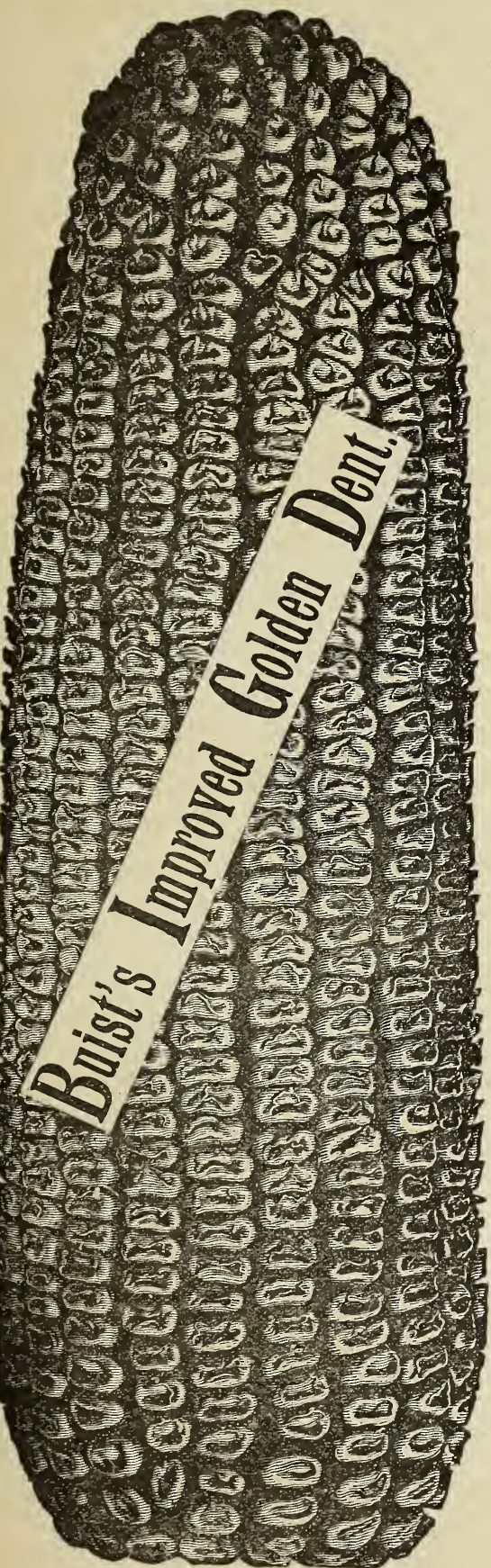

BUIST'S IMPROVED EARE " GOLDEN DENT CORN

The Best Yellow Field Corn for the South
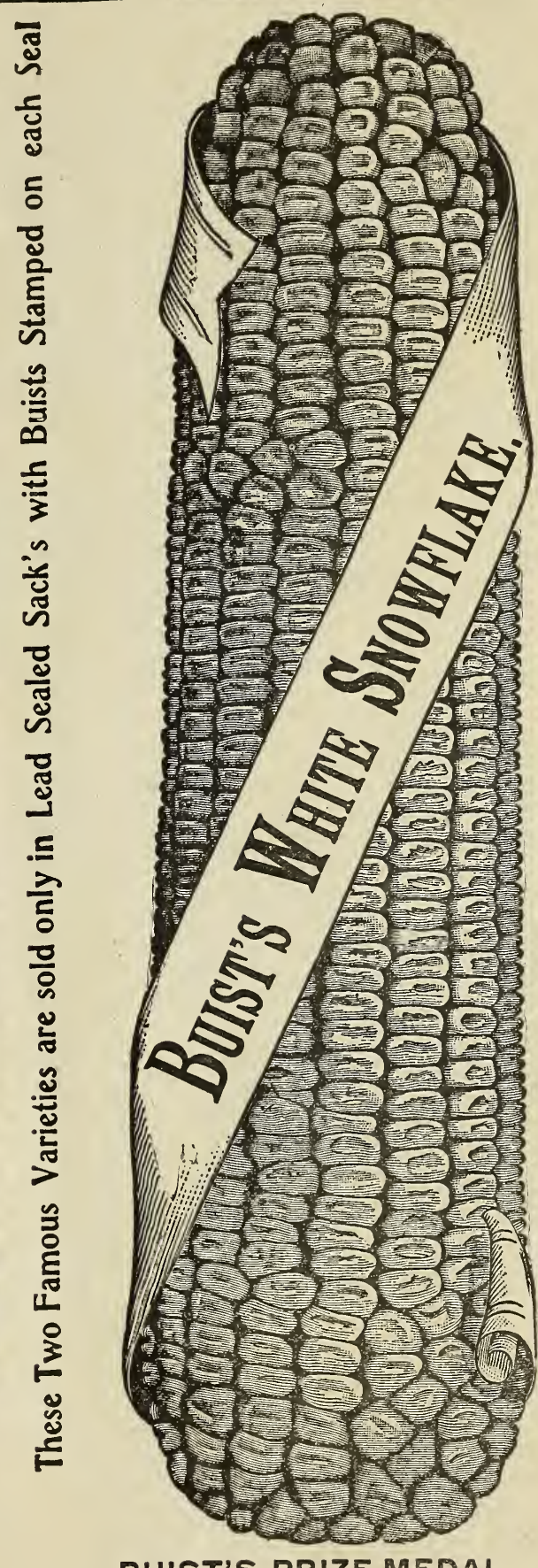

BUIST'S PRIZE MEDAL EARLY WHITE SNOW-FLAKE CORN The Best White Field Corn for the South It is also famous for producing the finest roasting ears.

Price Per Bushel . . . $\$ 1.50$ Price Per Bushel 
order ho.

ESTABLISHED IN 1828.

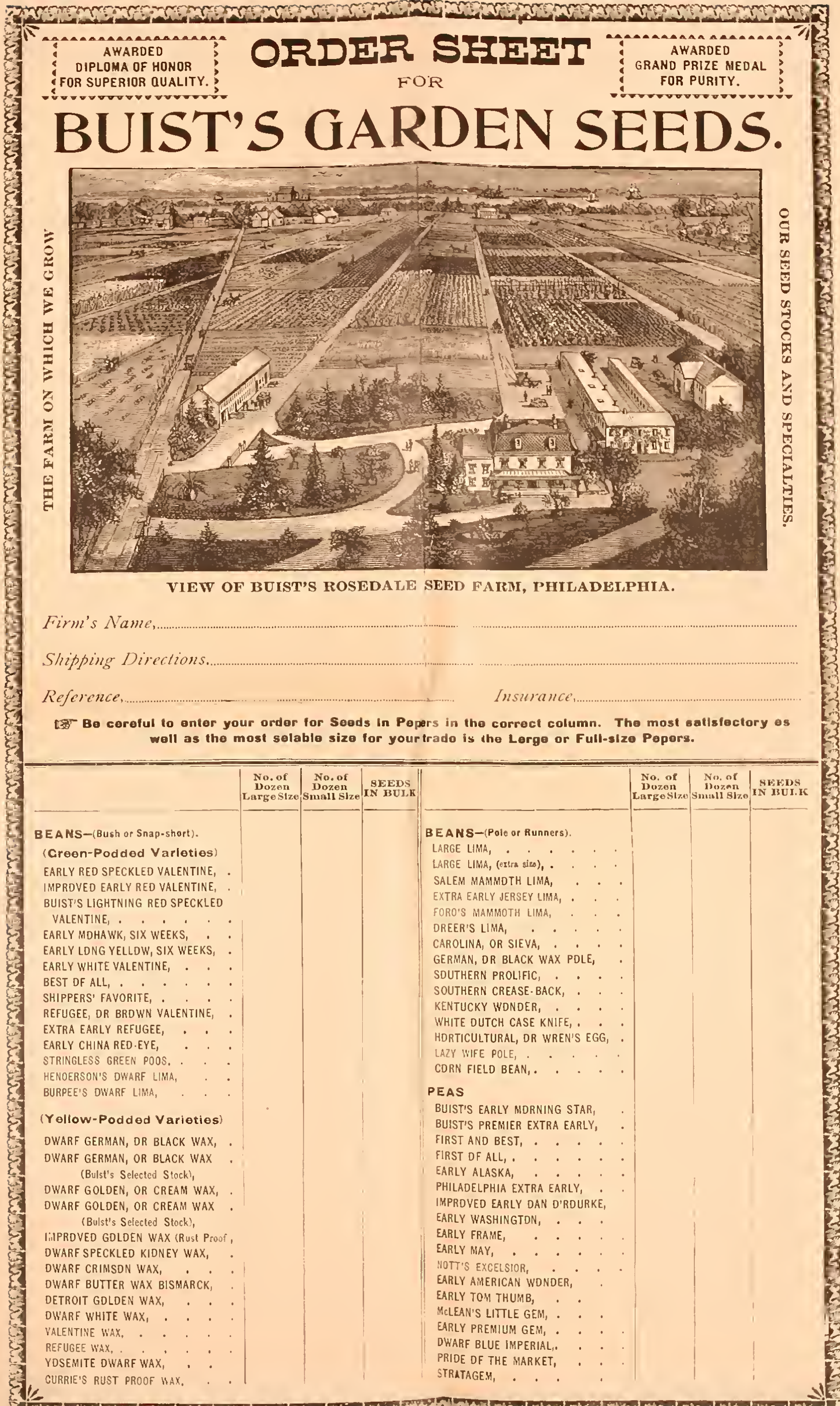




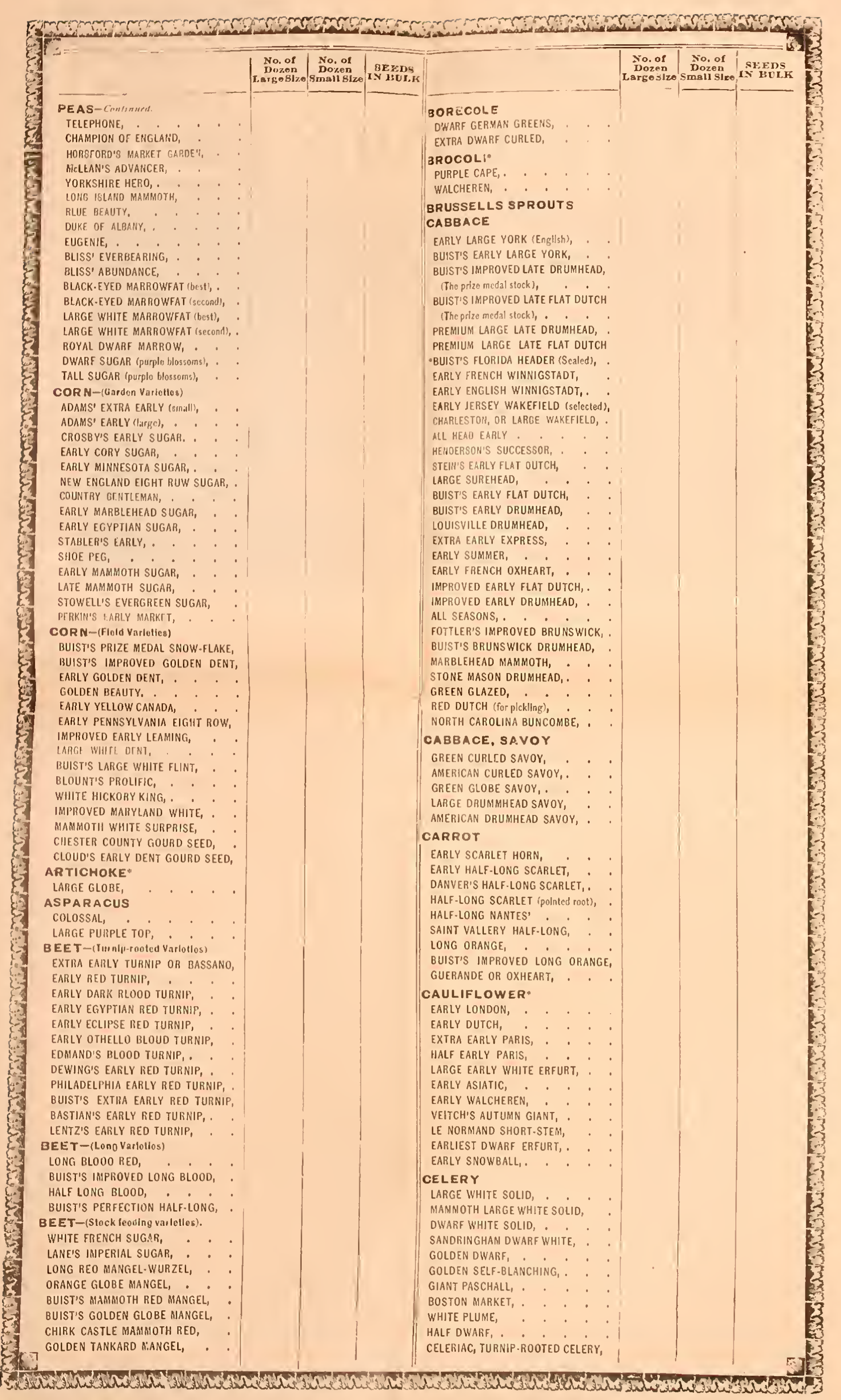

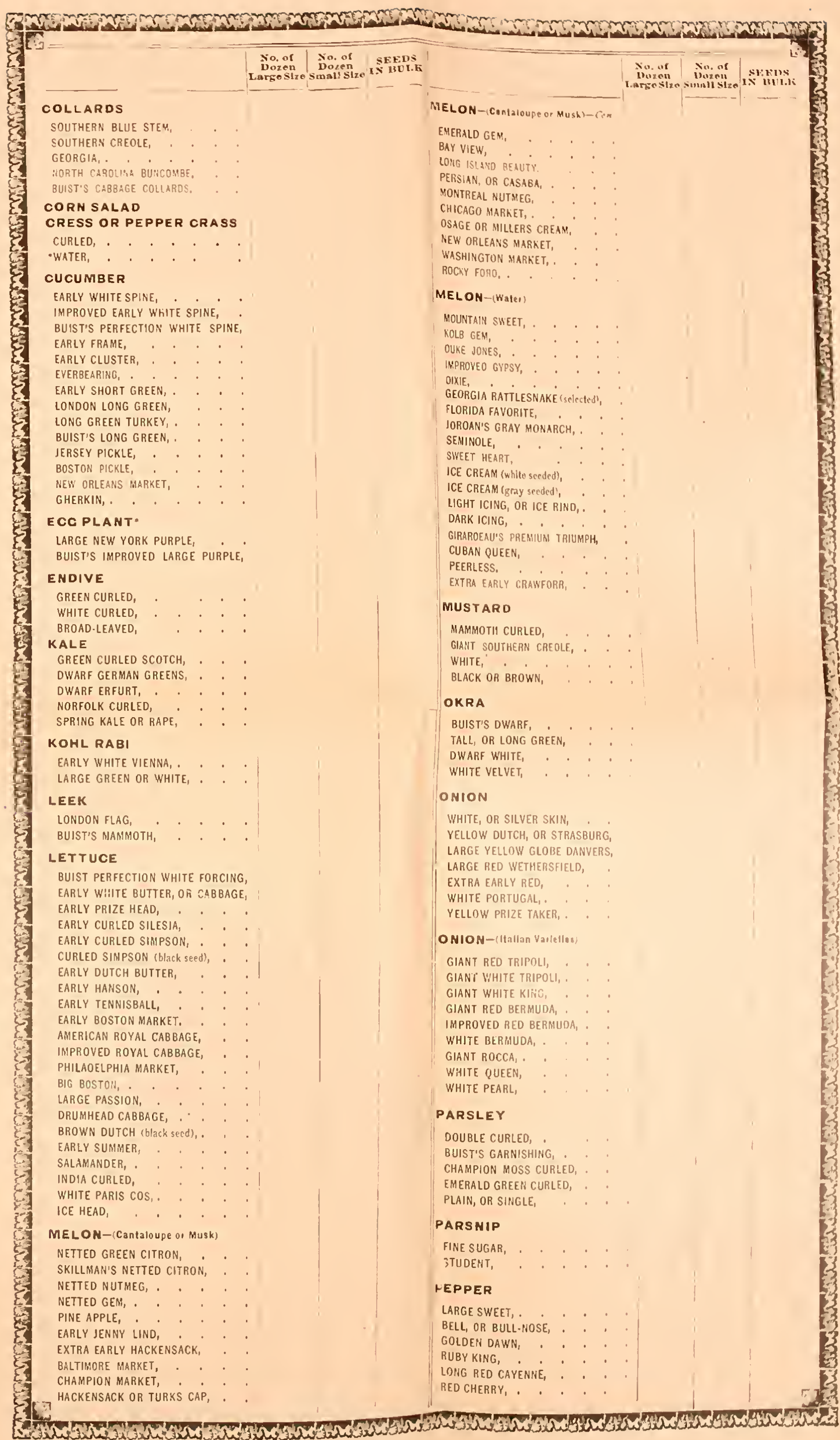



